\title{
BROMATOLOGÍA DE ENSILADOS DE CORONA DE PIÑA CON PULPA DE CÍTRICOS, HENO Y UREA ${ }^{1}$
}

\author{
Michael López-Herrera², Rodolfo WingChing-Jones ${ }^{2}$, Augusto Rojas-Bourrillon ${ }^{2}$
}

\begin{abstract}
RESUMEN
Bromatología de ensilados de corona de piña con pulpa de cítricos, heno y urea. El objetivo de esta investigación fue determinar las características nutricionales de los ensilados de mezclas de corona de la piña con inclusión de pulpa de cítricos deshidratada y heno, con niveles crecientes de urea. El experimento fue realizado entre los meses de mayo a setiembre del 2013 y las coronas provenían de Guápiles, Costa Rica. Estas fueron picadas hasta obtener una partícula promedio de $3 \mathrm{~cm}$, y colocadas en bolsas de empaque al vacío a las que se les agregó, urea $(0 ; 0,5 ; 1,0 ; 1,5 \%$ p/p en base fresca), dos fuentes altas en materia seca: heno $(\mathrm{H})$ de pasto Transvala (Digitaria decumbens, Stent., cv. Transvala) y pulpa de cítricos deshidratada (PCD) ambos a un nivel de inclusión ( $10 \% \mathrm{p} / \mathrm{p}$ en base fresca), y un único nivel de melaza $(3 \%$ $\mathrm{p} / \mathrm{p}$ ) e inóculo bacterial por fermentación de melaza con suero de leche $(1 \mathrm{l} / \mathrm{t})$. Además se preparó una muestra sin aditivos como control. El heno aumentó el contenido de materia seca y todos los componentes de la fibra, pero redujo el contenido de proteína cruda (PC), carbohidratos no fibrosos y nutrimentos digestibles totales. Cuando se usó PCD, se redujo la fracción fibrosa, lo que aumentó la digestibilidad de la mezcla ensilada. El uso de urea aumentó el contenido de PC y redujo el nivel de nitrógeno ligado a la fibra por un efecto de dilución. Se estimó que los materiales ensilados podrían producir 0,43-1,89 kg leche/vaca, al consumir $5 \mathrm{~kg}$ material verde (MV)/animal; 0,86-3,77 kg leche/vaca, al consumir $10 \mathrm{~kg}$ MV/animal; 1,29$5,66 \mathrm{~kg}$ leche/vaca, al consumir $15 \mathrm{~kg}$ MV/animal.
\end{abstract}

Palabras clave: conservación de forrajes, nutrición de rumiantes, subproductos piña, aditivos para piña, ensilaje.

\begin{abstract}
Bromatological composition of pineapple crown silage with citrus pulp, hay, and urea. The objective of this study was to determine the nutritional characteristics of silage mixtures of pineapple crown including dehydrated citrus pulp and hay, with increasing levels of urea. The experiment was conducted between May to September 2013 and the crowns were from Guapiles, Costa Rica. These were chopped to obtain an average particle size of $3 \mathrm{~cm}$, and packaged in vacuum- sealed bags to which were added: urea $(0,0.5,1.0$, $1.5 \% \mathrm{w} / \mathrm{w}$ fresh basis), 2 high sources of dry matter: hay $(\mathrm{H})$ of Transvala grass (Digitaria decumbens Stent, cv Transvala) and dehydrated citrus pulp (DCP), both at a level of inclusion (10\% w/w fresh basis), and a single level of molasses $(3 \%$ $\mathrm{w} / \mathrm{w}$ ) and bacterial inoculum by molasses fermentation with whey $(1 / / t)$. Also a sample without additives was prepared as a control. Hay increased dry matter content and all fiber components, but reduced crude protein content, non fibrous carbohydrates, and total digestible nutrients.. When PCD was used, the fiber level was reduced which increased digestibility of ensiled mixture. The use of urea increased the CP content and reduced nitrogen linked to fiber by a dilution effect. It was estimated that, silage materials allow to obtain from 0.43 to $1.89 \mathrm{~kg}$ of milk/cow, whenconsuming $5 \mathrm{~kg}$ fresh matter (FM)/animal; 0.86 to $3.77 \mathrm{~kg}$ milk/cow when consuming 10 $\mathrm{kg} \mathrm{FM} / \mathrm{animal} ; 1.29$ to $5.66 \mathrm{~kg}$ milk/cow when consuming $15 \mathrm{~kg} \mathrm{FM/animal.}$
\end{abstract}

Keywords: forage preservation, ruminant nutrition, pineapple byproducts, pineapple additives, silage.

1 Recibido: 27 de octubre, 2014. Aceptado: 26 de junio, 2015. Este trabajo forma parte del proyecto no. 739-B0-087 de la Vicerrectoría de Investigación de la Universidad de Costa Rica, San José, Costa Rica.

2 Universidad de Costa Rica, Escuela de Zootecnia, Centro de Investigación en Nutrición Animal. San José, Costa Rica. michael.lopez@ucr. ac.cr, rodolfo.wingching@ucr.ac.cr, augusto.rojas@ucr.ac.cr 


\section{INTRODUCCIÓN}

Los desechos del cultivo de la piña (Ananas comosus) generan forraje verde que puede ser aprovechable en la alimentación de rumiantes; en este sentido, las coronas representan entre el 9 y $11 \%$ del peso del fruto $(190-260 \mathrm{~g})$ de acuerdo con la densidad de siembra y cultivar (Rebolledo et al., 2006). Según trabajos realizados en cáscaras y pulpa (Gutiérrez et al., 2003), y en rastrojo de piña (López et al., 2009; Rodríguez-Chacón et al., 2014), los subproductos de esta industria pueden ser conservados de manera exitosa mediante la técnica del ensilaje.

Trabajos realizados en Brasil con rastrojo de piña (Cunha et al., 2009) y con cáscara y pulpa (Azevêdo et al., 2011), apuntan a que los subproductos de la industria de la piña pueden ser utilizados, ya sea en fresco o deshidratados (Rogério et al., 2007), en la alimentación de rumiantes. Estos materiales se consideran bajos en materia seca (MS), proteína cruda (PC) y grasas (Pereira et al., 2009); poseen altos contenidos de carbohidratos, tanto fibrosos como no fibrosos (Azevêdo et al., 2011) y un contenido energético similar a forrajes de uso en el trópico (López et al., 2009; Pereira et al., 2009).

Los subproductos de la piña, sobre todo la planta entera, coronas y rastrojos, se consideran bajos en materia seca $(\mathrm{MS})(7-13 \%)$ y proteína cruda (PC) (7,5-11\% MS). Sin embargo, poseen altos contenidos de carbohidratos, tanto fibrosos (FDN $=53-60 \% \mathrm{MS}$ ) como no fibrosos $(\mathrm{CNF}=21-24 \% \mathrm{MS})$ (Azevêdo et al., 2011; López et al., 2014) y un contenido energético $(\mathrm{NDT}=62-66 \%)$ similar o superior a forrajes de uso en el trópico (Pereira et al., 2009; López et al., 2014).

Al analizar las deficiencias que presentan los subproductos de piña, el uso de fuentes de nitrógeno no proteico podría disminuir el problema de bajo contenido de proteína cruda en el ensilado. Experiencias en Costa Rica, han demostrado que se puede incrementar el contenido de PC de los ensilados, elaborados con subproductos de piña, al incorporar nitrógeno no proteico en la mezcla, como por ejemplo Rodríguez-Chacón et al. (2014) y López et al. (2014) quienes incorporaron urea con rastrojos de piña, y Gutiérrez et al. (2003) que adicionaron pollinaza con desechos de piña como fuentes de nitrógeno. La metodología de incorporar una fuente de nitrógeno no proteico en ensilados elaborados con subproductos agrícolas con altos contenidos de carbohidratos ha ayudado a la mejoría de los mismos, y de la dieta de los animales que los consumen, así lo demostraron Arroyo et al. (2003) en dietas que contenían ensilados de pulpa de pejibaye (Bactris gasipaes Kunth).

El contenido de MS de rastrojos de piña se ha mejorado al utilizar pulpa de cítricos deshidratada (PCD) (López et al., 2009). También se ha propuesto el heno como un adecuado aditivo para aumentar la MS de los ensilajes (McDonald, 1981). El objetivo de esta investigación fue determinar las características nutricionales de los ensilados de mezclas de corona de la piña con inclusión de pulpa de cítricos deshidratada $\mathrm{y}$ heno, con niveles crecientes de urea.

\section{MATERIALES Y MÉTODOS}

El experimento se llevó a cabo entre mayo y setiembre del 2013 en un laboratorio de la Escuela de Zootecnia de la Universidad de Costa Rica, situado a 1205 msnm, en San Pedro de Montes de Oca, San José, Costa Rica. Las condiciones ambientales del laboratorio al momento del experimento fueron, temperatura promedio de $25^{\circ} \mathrm{C}$ y humedad relativa $>75 \%$.

Las coronas de piña utilizadas fueron recolectadas en Finca Babilonia, Guácimo, Limón, Costa Rica; la zona está a $268 \mathrm{msnm}$, presenta precipitaciones anuales entre $3000-4500 \mathrm{~mm}$; además, posee una temperatura promedio de $25{ }^{\circ} \mathrm{C}$. Las coronas se colectaron al azar de una pila de material fresco y triturados ese mismo día con cuchillo, hasta obtener un tamaño de partícula promedio de $3 \mathrm{~cm}$.

El experimento constó de ocho tratamientos en un arreglo factorial $(4 \times 2)$ con cuatro niveles de urea (U) $(0 ; 0,5 ; 1,0$ y $1,5 \% \mathrm{p} / \mathrm{p}$ en base fresca), dos alternativas de material deshidratante, heno $(\mathrm{H}) \mathrm{de}$ pasto Transvala (Digitaria decumbens, Stent., cv. Transvala) y pulpa de cítricos deshidratada (PCD), ambos con un nivel de inclusión de $10 \%$ p/p en base fresca. Todos los tratamientos poseían un único nivel de melaza $(3 \% \mathrm{p} / \mathrm{p})$ e inóculo bacterial artesanal elaborado por fermentación de melaza con suero de leche $(1 \mathrm{l} / \mathrm{t})$. Además, se preparó un ensilaje de coronas de piña sin aditivos, el cual se consideró como tratamiento control. Para la conservación del material se utilizaron bolsas de polietileno transparente para empaque al vacío con capacidad de $1 \mathrm{~kg}$ y con un 
grosor de $0,0063 \mathrm{~mm}$; cada bolsa se consideró como una unidad experimental. Cada tratamiento contó con cinco repeticiones para un total de 45 microsilos.

De cada tratamiento se tomó una muestra, previo al proceso de ensilaje; estas se llevaron al laboratorio de bromatología del Centro de Investigación en Nutrición Animal (CINA) de la Universidad de Costa Rica para analizar su composición al día 0 . El resto del material fue embolsado (45 microsilos) para someterlo al proceso de ensilaje; para tal fin, se utilizó una bomba de succión que extrajo el aire de las bolsas y así favorecer un proceso anaeróbico. Los microsilos se mantuvieron en condiciones ambientales (temperatura de $25{ }^{\circ} \mathrm{C}$ y humedad relativa $>80 \%$ ) por sesenta días.

Una vez finalizado el proceso de ensilaje, las mezclas fueron llevadas al CINA para analizarlas. Tanto a las muestras previas como a las ensiladas se les realizaron análisis de materia seca (MS), proteína cruda (PC), extracto etéreo (EE) y cenizas, de acuerdo con el manual de métodos oficiales de la AOAC (1998); los carbohidratos no fibrosos (CNF) se determinaron según la metodología de Detmann y Filho (2010). En el caso de la fibra detergente neutro (FDN), fibra detergente ácido (FDA), hemicelulosa (HEM), celulosa (CEL) y lignina (LIG), se procedió con la metodología descrita por Van Soest y Robertson (1985). Para la estimación de los nutrimentos digestibles totales (NDT) se utilizaron las ecuaciones descritas por Weiss (2004); para la estimación de energía se utilizaron las ecuaciones del NRC (2001). La determinación de la proteína insoluble en FDN (PIDN) y la proteína insoluble en FDA (PIDA) se realizó utilizando los procedimientos descritos en Licitra et al. (1996).

Se calculó el aporte de la PC y la energía neta de lactación $\left(\mathrm{EN}_{\mathrm{L}}\right)$ de cada una de las mezclas ensiladas, con base en tres niveles de consumo de material verde fresco (MV) (5, 10 y $15 \mathrm{~kg} \mathrm{MV/vaca/día)} \mathrm{de} \mathrm{acuerdo} \mathrm{con}$ lo descrito por Sánchez (2010); los resultados obtenidos fueron comparados con los requerimientos para la producción de leche de una vaca de $454 \mathrm{~kg}$, publicados en los cuadros de requerimientos del NRC (2001).

Para el análisis de la información se utilizó un modelo de análisis de varianza de INFOSTAT (Di Rienzo et al., 2013), de acuerdo con el siguiente modelo estadístico:

$$
\mathrm{y}_{\mathrm{ijk}}=\mu+\mathrm{U}_{\mathrm{i}}+\mathrm{S}_{\mathrm{j}}+(\mathrm{MxU})_{\mathrm{ij}}+\varepsilon_{\mathrm{ijk}}
$$

Donde:

y = variable de respuesta obtenida de la ecuación.

$\mathrm{m}=$ media general.

$\mathrm{U}=$ efecto i ésimo de la urea en los tratamientos.

$\mathrm{S}=$ efecto $\mathrm{j}$ ésimo del material secante en los tratamientos.

$\mathrm{SxU}=$ efecto ij ésimo de la interacción entre los aditivos urea y el material secante.

$\varepsilon=$ término de error, donde $\varepsilon \sim \mathrm{N}\left(0, \sigma^{2}\right)$.

Para determinar la significancia de los efectos principales se realizó, la comparación entre medias mediante la prueba de Tukey con un nivel de 0,05.

\section{RESULTADOS Y DISCUSIÓN}

Materia seca. El contenido de MS de los tratamientos se vio afectado de manera significativa $(\mathrm{p}<0,05)$ por la inclusión de heno y PCD; no obstante, la inclusión de urea no afectó esta variable (Cuadro 1). El contenido de MS de las coronas sin aditivos fue bajo, debido a estructuras en las hojas de la planta y las coronas donde se almacenó el agua (Bartholomew et al., 2003); además, ocurre una acumulación de agua por la técnica utilizada (microsilos) de acuerdo con lo concluido por López et al. (2009).

En el caso de los tratamientos donde se adicionó heno y PCD, se dio un incremento en el contenido de MS conforme se aumentó el porcentaje de inclusión de estos materiales, este incremento fue similar al obtenido por Gutiérrez et al. (2003) y López et al. (2009) quienes trabajaron con pollinaza y la PCD, respectivamente.

El contenido de MS en todos los tratamientos fue menor que el obtenido por Lallo et al. (2003) (14,78\%) y Kellems et al. (1979) $(22,52 \%)$ en planta entera con cáscaras de piña. Por otra parte, experimentos (Cunha et al., 2009; Azevêdo et al., 2011) con rastrojos $(13,91 \%)$, y con ensilados de cáscara y pulpa $(13,4 \%)$, mostraron resultados similares a los obtenidos en los tratamientos con adición de heno y PCD, aunque el tratamiento control presentó contenidos menores de MS. Los tratamientos con 10\% de inclusión de PCD presentaron contenidos menores de MS con respecto a los valores presentados por López et al. (2009), lo cual 
Cuadro 1. Calidad nutricional (\% MS) de las mezclas de coronas de piña con adición de heno y urea, 60 días después del proceso de ensilaje. San José, Costa Rica. 2014.

Table 1. Nutritional value (\%DM) of pineapple crown silage mixtures with hay and urea, after 60 days of storage. San José, Costa Rica. 2014.

\begin{tabular}{lccccccccc}
\hline & & \multicolumn{3}{c}{ Pulpa de cítricos deshidratada (10\% p/pcpp) } & \multicolumn{3}{c}{ Heno (10\% p/p) } \\
\cline { 3 - 10 } & & \multicolumn{3}{c}{ Nivel de urea $\mathbf{\%}$ p/p) } & \multicolumn{3}{c}{ Nivel de urea (\% p/p) } \\
\cline { 3 - 10 } Variables* & Control & $\mathbf{0}$ & $\mathbf{0 , 5}$ & $\mathbf{1}$ & $\mathbf{1 , 5}$ & $\mathbf{0}$ & $\mathbf{0 , 5}$ & $\mathbf{1}$ & $\mathbf{1 , 5}$ \\
\hline MS (\%) & $7,76 \mathrm{a}$ & $13,54 \mathrm{~b}$ & $13,95 \mathrm{~b}$ & $14,39 \mathrm{~b}$ & $13,76 \mathrm{~b}$ & $13,89 \mathrm{~b}$ & $15,51 \mathrm{~b}$ & $13,45 \mathrm{~b}$ & $13,57 \mathrm{~b}$ \\
PC (\% MS) & $12,94 \mathrm{ab}$ & $12,63 \mathrm{ab}$ & $17,26 \mathrm{c}$ & $23,99 \mathrm{~d}$ & $32,06 \mathrm{e}$ & $9,65 \mathrm{a}$ & $14,81 \mathrm{bc}$ & $25,36 \mathrm{~d}$ & $31,98 \mathrm{e}$ \\
PIDN (\% PC) & $13,52 \mathrm{~cd}$ & $16,86 \mathrm{de}$ & $13,04 \mathrm{bcd}$ & $9,38 \mathrm{abc}$ & $7,02 \mathrm{a}$ & $21,35 \mathrm{e}$ & $16,88 \mathrm{de}$ & $9,38 \mathrm{abc}$ & $8,01 \mathrm{ab}$ \\
PIDA (\% PC) & $9,66 \mathrm{~cd}$ & $11,88 \mathrm{~d}$ & $9,04 \mathrm{c}$ & $6,00 \mathrm{ab}$ & $4,49 \mathrm{a}$ & $11,71 \mathrm{~d}$ & $7,63 \mathrm{bc}$ & $4,93 \mathrm{a}$ & $3,72 \mathrm{a}$ \\
EE (\% MS) & $4,01 \mathrm{abc}$ & $6,34 \mathrm{~d}$ & $2,69 \mathrm{a}$ & $5,28 \mathrm{bcd}$ & $4,51 \mathrm{abcd}$ & $3,35 \mathrm{ab}$ & $3,64 \mathrm{ab}$ & $4,47 \mathrm{abcd}$ & $5,72 \mathrm{~cd}$ \\
FDN (\% MS) & $62,41 \mathrm{bc}$ & $51,20 \mathrm{a}$ & $48,27 \mathrm{a}$ & $46,86 \mathrm{a}$ & $48,84 \mathrm{a}$ & $70,84 \mathrm{~cd}$ & $71,06 \mathrm{~d}$ & $65,34 \mathrm{bcd}$ & $62,29 \mathrm{~b}$ \\
FDA (\% MS) & $37,64 \mathrm{~cd}$ & $31,69 \mathrm{ab}$ & $29,97 \mathrm{a}$ & $29,39 \mathrm{a}$ & $30,14 \mathrm{a}$ & $40,46 \mathrm{~d}$ & $39,68 \mathrm{~cd}$ & $37,00 \mathrm{~cd}$ & $35,18 \mathrm{bc}$ \\
HEM (\% MS) & $24,77 \mathrm{~b}$ & $19,51 \mathrm{a}$ & $18,31 \mathrm{a}$ & $17,47 \mathrm{a}$ & $18,70 \mathrm{a}$ & $30,38 \mathrm{c}$ & $31,38 \mathrm{c}$ & $28,34 \mathrm{bc}$ & $27,10 \mathrm{bc}$ \\
CEL (\% MS) & $35,88 \mathrm{~cd}$ & $29,82 \mathrm{ab}$ & $28,05 \mathrm{a}$ & $27,55 \mathrm{a}$ & $28,38 \mathrm{ab}$ & $37,85 \mathrm{~d}$ & $37,00 \mathrm{~cd}$ & $34,53 \mathrm{~cd}$ & $32,72 \mathrm{bc}$ \\
LIG(\% MS) & $1,76 \mathrm{a}$ & $1,87 \mathrm{a}$ & $1,92 \mathrm{a}$ & $1,84 \mathrm{a}$ & $1,76 \mathrm{a}$ & $2,61 \mathrm{~b}$ & $2,68 \mathrm{~b}$ & $2,48 \mathrm{~b}$ & $2,46 \mathrm{~b}$ \\
CNF (\% MS)** & $14,83 \mathrm{ab}$ & $23,21 \mathrm{bc}$ & $30,54 \mathrm{c}$ & $28,11 \mathrm{c}$ & $24,17 \mathrm{c}$ & $8,70 \mathrm{a}$ & $9,40 \mathrm{a}$ & $9,01 \mathrm{a}$ & $9,79 \mathrm{a}$ \\
Cenizas (\% MS) & $7,58 \mathrm{a}$ & $8,73 \mathrm{bc}$ & $8,67 \mathrm{bc}$ & $8,32 \mathrm{ab}$ & $7,80 \mathrm{a}$ & $9,53 \mathrm{~d}$ & $9,17 \mathrm{~cd}$ & $9,02 \mathrm{bcd}$ & $8,69 \mathrm{bc}$ \\
\hline
\end{tabular}

Valores en la misma fila con diferentes letras son significativamente diferentes $(\mathrm{p}<0,05)$ / Values in the same row with different letters are significantly different $(\mathrm{p}<0.05)$.

* Materia seca (MS), proteína cruda (PC), extracto etéreo (EE), carbohidratos no fibrosos (CNF) fibra detergente neutro (FDN), fibra detergente ácido (FDA), hemicelulosa (HEM), celulosa (CEL), lignina (LIG), proteína insoluble en solución detergente neutro (PIDN), proteína insoluble en solución detergente ácida (PIDA) / Dry matter (DM), crude protein (CP), ether extract (EE), non-fibrous carbohydrates (NFC) neutral detergent fiber (NDF), acid detergent fiber (ADF), hemicellulose (HEM), cellulose (CEL), lignin (LIG), neutral detergent insoluble protein (NDIP), acid detergent insoluble protein (ADIP).

** Corregido para eliminar el efecto de la urea en la estimación de los CNF en la mezcla, de acuerdo a Detmann y Filho (2010) / Corrected to eliminate the effect of urea in the estimation of NFC of silage mixtures, according to Detmann y Filho (2010).

se podría relacionar con el material utilizado, donde las coronas de piña presentaron un menor contenido de MS con respecto a los rastrojos, utilizados en la investigación de los autores mencionados.

De acuerdo con los requerimientos del NRC (2001), una vaca de $454 \mathrm{~kg}$ de peso corporal en lactancia media, que produce $10 \mathrm{~kg}$ de leche con 4\% grasa/día, debe consumir 12,4 kg MS/día con una dieta menor al $68 \%$ de NDT. De acuerdo con la ecuación propuesta por Belyea et al. (1996), la cual relaciona la FDN con el consumo de MS, los tratamientos evaluados fluctuaron entre 1,68-2,56\% del peso corporal; es decir, que los ensilados podrían proveer entre 7,62 - 11,62 kg de MS respectivamente, siendo los tratamientos con inclusión de PCD los que permiten mayor consumo de MS y los de inclusión de heno los que limitan la capacidad de consumo en el animal (Sánchez y Soto, 1998). Por lo tanto, los ensilados evaluados en este experimento no pueden ser utilizados como único alimento en una dieta de vacas en producción, debido a que no llenan los requerimientos de MS, por lo que deben ser utilizados como parte de una ración total.

Proteína cruda. La interacción entre el material secante y la urea afectaron el contenido de PC en las mezclas (Cuadro 1), siendo las diferencias entre los tratamientos significativas $(\mathrm{p}<0,05)$; este efecto podría relacionarse con el bajo contenido de $\mathrm{PC}$ de la PCD (López et al., 2009) y del heno (WingChing y Alvarado, 2009). Por otra parte, el incremento en la 
concentración de urea aumentó de forma proporcional el contenido de PC en los ensilados (Cuadro 1), el mismo comportamiento se encontró en ensilados de rastrojo de piña (López et al., 2014); esto concuerda con lo descrito por McDonald (1981) quien afirma que, al utilizar urea en ensilajes, se incrementa el contenido de proteína cruda y verdadera, aminoácidos libres y amoniaco, ya que ocurre mayor síntesis de proteína microbiana, principalmente aminoácidos como: alanina, ácido aspártico, ácido glutámico, isoleucina, lisina, treonina y valina.

Los ensilados evaluados tuvieron un contenido de PC que fue mayor que los valores presentados por López et al. (2009) $(7,08-8,24 \%$ MS), Prado et al. (2003) (8,83\% MS), Azevêdo et al. (2011) (7,09\% MS) y Correia et al. (2006) (7,37\% MS), debido a que las coronas poseen un mayor contenido de PC que otros subproductos de la piña (rastrojos, cáscaras, cáscara y pulpa, respectivamente); además, la inclusión de urea acentúa esta diferencia. Los tratamientos con niveles de 1 y $1,5 \% \mathrm{p} / \mathrm{p}$ de urea presentaron niveles mayores de PC con respecto a los valores obtenidos en coronas de piña (Rodríguez-Chacón et al., 2014), lo cual favorece el uso de aditivos para la conservación de las coronas de piña y su eventual suministro en la dieta de animales rumiantes.
Los materiales ensilados presentaron contenidos de PIDN y de PIDA menores a los obtenidos por Lousada Jr et al. (2005) (38,4 y 16,35 PC) en pulpa de la fruta, Pereira et al. (2009) $(55,61-26,02 \%$ PC) en cáscara y pulpa del fruto y Azevêdo et al. (2011) en rastrojo (50,49 y $44,38 \%$ PC). Por otra parte, los resultados obtenidos fueron similares a los reportados en planta entera $(10,81$ $-8,43 \%$ PC) (Rogério et al., 2007). La inclusión de PCD y urea no afectó de forma significativa los contenidos de PIDN y PIDA (Cuadro 1); sin embargo, se detectó una tendencia al aumento de las fracciones nitrogenadas ligadas a la FDN y FDA cuando se utilizó PCD, lo cual se podría relacionar con daños de la proteína durante el proceso de deshidratación. La inclusión de heno aumentó estas fracciones de la PC en el ensilaje de corona de piña, ya que este material posee alto contenido de PIDN (40,2\% PC) y PIDA (49,3\% PC) (WingChing y Alvarado, 2009). También se determinó que al incrementar la concentración de urea en la mezcla ensilada, se redujeron los contenidos de PIDN y PIDA, esto debido a un aumento en el contenido de PC por aumento en uso de NNP, lo que provocó un efecto de dilución que disminuyó la concentración de estas fracciones (Cuadro 1).

En la Figura 1 se observa el potencial, en términos de aporte de proteína, que tienen los materiales

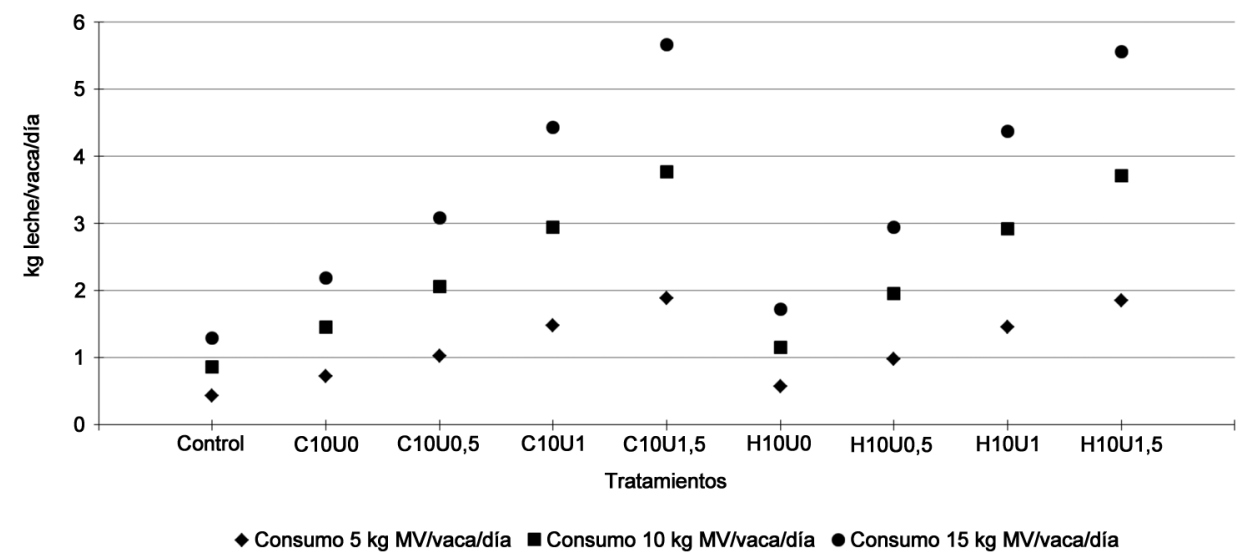

Figura 1. Potencial de producción (por requerimiento de proteína) en kg de leche/vaca/día, de los ensilados de corona de piña con pulpa de cítricos deshidratada (C), heno (H) y urea (U) de acuerdo al nivel de consumo de materia verde (MV) (5, 10 y $15 \mathrm{~kg}$ MV/día). San José, Costa Rica. 2014.

Figure 1. Potential production (by protein requirement) in $\mathrm{kg}$ of milk/cow/day, from pineapple crown silage with dehydrated citrus pulp (C), hay (H), and urea (U), according to fresh matter (FM) intake (5, 10, and $15 \mathrm{~kg} \mathrm{FM/day).} \mathrm{San} \mathrm{José,} \mathrm{Costa} \mathrm{Rica.} 2014$. 
evaluados para la producción de leche. En un estudio se consideró posible utilizar hasta $15 \mathrm{~kg}$ de corona fresca en la alimentación de vacas lecheras sin sacrificar su producción (Sánchez, 2010). Basados en el aporte de proteína de los ensilados, se observó que tanto los tratamientos con heno como los tratamientos con PCD, permitirían producir entre $2-5,5 \mathrm{~kg}$ leche/ vaca/día, cuando este animal consume $15 \mathrm{~kg} \mathrm{MV/}$ vaca/día y no más de $2 \mathrm{~kg}$ leche/vaca/día, cuando el consumo es de $5 \mathrm{~kg} \mathrm{MV/vaca/día.} \mathrm{Las} \mathrm{diferencias}$ entre los tratamientos dependen de la adición de urea en la mezcla ensilada, ya que este aditivo aumenta la cantidad de proteína degradable en el rumen al ser fuente de nitrógeno no proteico (NNP).

Extracto etéreo. Ninguno de los aditivos utilizados en este trabajo mostró diferencias significativas entre los tratamientos evaluados (Cuadro 2). La concentración de EE en los ensilados obtenidos en esta investigación fueron mayores a los publicados por López et al. (2009) (1,56-2,24\% MS), Gutiérrez et al. (2003) (1,58 - 2,52\% MS), Lousada Jr et al. (2005) (1,2\% MS), Cunha et al. (2009) $(2,20-2,42 \%$ MS), Pereira et al. (2009) (0,60\% MS) y Rodríguez-Chacón et al. (2014) (1,6-3,2\% MS), en diferentes partes de la planta; lo que podría explicar las diferencias en el contenido de extracto etéreo.

Cenizas. En cuanto a la fracción mineral, tanto la PCD como el heno mostraron diferencias significativas $(\mathrm{p}<0,05)$ con respecto al tratamiento control, ya que al aumentar el contenido de heno se tiende a generar un incremento en el contenido de materiales (Cuadro 1). El uso de urea redujo el contenido mineral conforme se incrementó la inclusión de la misma, este efecto fue opuesto al encontrado por Rodríguez-Chacón et al. (2014), quienes indicaron que el uso de urea tiende a elevar la concentración de cenizas en la muestra.

Los valores obtenidos en los tratamientos sin heno fueron menores a los valores publicados por Pereira et al. (2009) (10,70\% MS) en cáscara y pulpa del fruto y Rogério et al. (2007) $(9,20 \%$ MS) en plantas enteras esta diferencia se puede deber a que en estos ensilados se incluyeron partes de la planta como la raíz o se han deshidratado, proceso que tiende a concentrar los nutrimentos en los forrajes. En el caso de los tratamientos con adición de heno, estos presentaron valores menores a los descritos por Lallo et al. (2003) (17,02\% MS) en cáscaras del fruto, pero similares a los obtenidos por Correia et al. (2006) (10,08\% MS) en cáscara y pulpa del

Cuadro 2. Contenido energético en base seca de los ensilados de corona de piña con adición de PCD, heno y urea. San José, Costa Rica. 2014.

Table 2. Energy value in dry matter of pineapple crown silage with dehydrated citrus pulp, hay, and urea. San José, Costa Rica. 2014.

\begin{tabular}{|c|c|c|c|c|c|c|c|c|c|}
\hline \multirow[b]{3}{*}{ Variables* } & \multirow[b]{3}{*}{ Control } & \multicolumn{4}{|c|}{ Pulpa de cítricos deshidratada $(10 \%$ p/p) } & \multicolumn{4}{|c|}{ Heno $(10 \%$ p/p) } \\
\hline & & \multicolumn{4}{|c|}{ Nivel de urea $(\% \mathrm{p} / \mathrm{p})$} & \multicolumn{4}{|c|}{ Nivel de urea $(\% \mathrm{p} / \mathrm{p})$} \\
\hline & & $\mathbf{0}$ & 0,5 & 1 & 1,5 & $\mathbf{0}$ & 0,5 & 1 & 1,5 \\
\hline NDT $(\%)$ & $62,05 \mathrm{ab}$ & $65,97 \mathrm{~cd}$ & $67,10 \mathrm{~d}$ & $67,48 \mathrm{~d}$ & $66,99 \mathrm{~d}$ & $60,19 \mathrm{a}$ & $60,71 \mathrm{ab}$ & $62,47 \mathrm{ab}$ & $63,67 \mathrm{bc}$ \\
\hline ED (Mcal kg MS) & $2,74 \mathrm{ab}$ & $2,91 \mathrm{~cd}$ & $2,96 \mathrm{~d}$ & $2,98 \mathrm{~d}$ & $2,95 \mathrm{~d}$ & $2,65 \mathrm{a}$ & $2,68 a b$ & $2,75 \mathrm{ab}$ & $2,81 \mathrm{bc}$ \\
\hline EM (Mcal kg MS) & $2,24 \mathrm{ab}$ & $2,39 \mathrm{~cd}$ & $2,43 \mathrm{~d}$ & $2,44 \mathrm{~d}$ & $2,42 \mathrm{~d}$ & 2,18 a & $2,19 \mathrm{ab}$ & $2,26 \mathrm{ab}$ & $2,30 \mathrm{bc}$ \\
\hline $\mathrm{EN}_{\mathrm{L}}(\mathrm{Mcal} \mathrm{kg} \mathrm{MS})$ & $1,40 \mathrm{ab}$ & $1,50 \mathrm{~cd}$ & $1,52 \mathrm{~d}$ & $1,53 \mathrm{~d}$ & $1,52 \mathrm{~d}$ & $1,35 \mathrm{a}$ & $1,37 \mathrm{ab}$ & $1,41 \mathrm{ab}$ & $1,44 \mathrm{bc}$ \\
\hline $\mathrm{EN}_{\mathrm{L}}(\mathrm{Mcal} \mathrm{kg} \mathrm{MS})(3 \mathrm{X})$ & $1,36 \mathrm{ab}$ & $1,42 \mathrm{~cd}$ & $1,44 \mathrm{~d}$ & $1,45 \mathrm{~d}$ & $1,44 \mathrm{~d}$ & $1,33 \mathrm{a}$ & $1,34 \mathrm{ab}$ & $1,37 \mathrm{ab}$ & $1,39 \mathrm{bc}$ \\
\hline $\mathrm{EN}_{\mathrm{M}}($ Mcal kg MS $)$ & $1,38 \mathrm{ab}$ & $1,50 \mathrm{~cd}$ & $1,54 \mathrm{~d}$ & $1,55 \mathrm{~d}$ & $1,54 \mathrm{~d}$ & $1,32 \mathrm{a}$ & $1,33 \mathrm{ab}$ & $1,39 \mathrm{ab}$ & $1,43 \mathrm{bc}$ \\
\hline $\mathrm{EN}_{\mathrm{G}}($ Mcal kg MS $)$ & $0,80 \mathrm{ab}$ & $0,91 \mathrm{~cd}$ & $0,94 \mathrm{~d}$ & $0,96 \mathrm{~d}$ & $0,94 \mathrm{~d}$ & $0,74 \mathrm{a}$ & $0,76 \mathrm{ab}$ & $0,81 \mathrm{ab}$ & $0,85 \mathrm{bc}$ \\
\hline
\end{tabular}

Valores en la misma fila con diferentes letras son significativamente diferentes $(p<0,05) /$ Values in the same row with different letters are significantly different $(\mathrm{p}<0.05)$.

*Nutrimentos digestibles totales (NDT), energía digestible (ED), energía metabolizable (EM), energía neta de lactación ( $\mathrm{EN}_{\mathrm{L}}$ ), energía neta de mantenimiento $\left(\mathrm{EN}_{\mathrm{M}}\right)$, energía neta de ganancia de peso $\left(\mathrm{EN}_{\mathrm{G}}\right)$ / Total digestible nutrients (TDN), digestible energy $(\mathrm{DE})$, metabolizable energy $(\mathrm{ME})$, net energy for lactation $\left(\mathrm{NE}_{\mathrm{L}}\right)$, net energy for maintenance $\left(\mathrm{NE}_{\mathrm{M}}\right)$, net energy for weight gain $\left(\mathrm{NE}_{\mathrm{G}}\right)$. 
fruto. Estas diferencias se podrían relacionar con el tipo de manejo en el cultivo y cultivar utilizado (Rebolledo et al., 2006); además, el proceso de deshidratación tiende a concentrar nutrimentos. El tratamiento control presentó contenido de cenizas similares a los resultados obtenidos por Cunha et al. (2009) $(4,8-6,05 \% \mathrm{MS})$.

Fibra detergente neutro. La inclusión de heno tendió a aumentar el contenido de FDN en los ensilados, pero las diferencias no fueron significativas; no obstante, la inclusión de PCD disminuyó el contenido de esta fracción en los materiales evaluados $(\mathrm{p}<0,05)$ (Cuadro 1). La urea también provocó diferencias significativas entre los tratamientos evaluados. Se encontró una tendencia a la reducción en el contenido de FDN cuando se incrementó el contenido de urea en la mezcla. Este efecto pudo haber sido provocado por una mayor actividad microbiana en el ensilaje, que aprovechó la urea y la fibra como fuente de energía, resultados que concuerdan con otros estudios (McDonald, 1981).

El contenido de FDN de los tratamientos con heno fue mayor a los valores de $41,8-56,0 \%$ MS reportados en rastrojos de piña con adición de PCD (López et al., 2009), a pesar de que del estudio los tratamientos con PCD evaluados fueron similares a los resultados del estudio mencionado. Además, existe similitud entre los resultados obtenidos en esta investigación y los resultados de los trabajos de Azevêdo et al. (2011) (63,77\% MS), Pereira et al. (2009) (60,30\% MS), Cunha et al. (2009) (55,31-56,6\% MS), Gutiérrez et al. (2003) (59,88 - 67,08\% MS), Rogério et al. (2007) $(66,14 \%$ MS). Mas no con los trabajos de Correia et al. (2006) $(72,12 \%$ MS) y Lousada Jr et al. (2005) (71,4\% MS), ya que estos presentaron valores mayores de esta fracción.

En el caso de las diferencias con otras investigaciones, estas podrían relacionarse con diferentes factores, tales como la parte de la planta (Kellems et al., 1979), el aditivo utilizado en el proceso de ensilaje (Gutiérrez et al., 2003), el proceso al que se sometió el material previo a su conservación (Correia et al., 2006; Rogério et al., 2007) y la variedad utilizada (Rebolledo et al., 2006).

Fibra detergente ácido. Los tratamientos evaluados presentaron diferencias significativas $(\mathrm{p}<0,05)$ al incorporar PCD y heno a la mezcla. Cuando se utilizó PCD, el contenido de FDA de los tratamientos se redujo; sin embargo, cuando se incorporó el heno, se incrementó, diferencias que se podrían deber al contenido de esta fracción en cada uno de los materiales (Cuadro 1). La tendencia obtenida con el heno y la FDA, podría indicar una menor digestibilidad, ya que se ha considerado que niveles de FDA altos se relacionan con niveles de digestibilidad bajos en forrajes (Van Soest et al., 1991; Sánchez y Soto, 1998).

Los tratamientos con inclusión de PCD presentaron contenidos de FDA mayores a los encontrados por López et al. (2009) $(27,3$ - 35,00\% MS), Cunha et al. (2009) (31,95 - 32,60\% MS) y Rogério et al. (2007) $(34,41 \%$ MS), pero menores a los descritos por Rodríguez-Chacón et al. (2014) (37,7 - 42,55\% MS), diferencias que podrían asociarse con la parte de la planta o subproducto evaluado. En el caso de los tratamientos con inclusión de heno, estos presentaron contenidos de esta fracción mayores a los obtenidos por Gutiérrez et al. (2003) (35,39 - 36,93\% MS), Azevêdo et al. (2011) (34,11\% MS), Lousada Jr et al. (2005) (30,7\% MS), debido a que el heno es un material alto en FDA (WingChing y Alvarado, 2009) y su inclusión eleva el contenido de esta fracción en la mezcla ensilada.

Hemicelulosa y celulosa. Tanto el heno como la PCD afectaron el contenido de hemicelulosa y celulosa de los materiales ensilados. Cuando se utilizó PCD se redujo el contenido de estas fracciones (CEL) en los tratamientos, debido a un menor contenido de estos nutrientes en el aditivo. De forma contraria, el uso de heno aumentó significativamente $(\mathrm{p}<0,05)$ el contenido de estas fracciones de fibra con respecto a las coronas ensiladas sin aditivos (Cuadro 1).

Los niveles crecientes de urea no afectaron el contenido de hemicelulosa y celulosa de forma significativa; sin embargo, se encontró una tendencia a la disminución del contenido de estas fracciones de la fibra conforme se incrementó el contenido de urea, lo cual se podría relacionar con el hecho de que los componentes de la pared celular pueden ser aprovechados por los microorganismos durante el proceso de ensilaje y que al incorporar urea en el silo es posible aumentar el crecimiento bacteriano, lo que potencia el aprovechamiento de los componentes de la FDN (McDonald, 1981).

Los valores obtenidos en esta investigación, para el tratamiento control, fueron mayores a los publicados por López et al. (2009) (HEM=14,1 - 21,0\% MS; $\mathrm{CEL}=23,6-32,2 \% \mathrm{MS}$ ) y Lousada Jr et al. (2005) 
$(\mathrm{HEM}=40,7 \% \mathrm{MS} ; \mathrm{CEL}=25,9 \% \mathrm{MS})$, pero menores a los informados por Correia et al. (2006) (HEM= $38,4 \%$ MS; CEL $=24,0 \%$ MS) y Rogério et al. (2007) (HEM $=31,7 \%$ MS; CEL $=37,7 \% \mathrm{MS}$ ), estas variantes en el contenido de hemicelulosa y celulosa, se podrían asociar con la parte de la planta evaluada (López et al., 2014).

Lignina. El contenido de esta fracción de la fibra presentó diferencias significativas $(\mathrm{p}<0,05)$ cuando se utilizó PCD y heno en la mezcla ensilada, ambos aditivos aumentaron el contenido de lignina en los ensilados, debido a que poseen mayor contenido de esta fracción con respecto a las coronas de piña (Cuadro 1). El contenido de lignina de los materiales se relacionó de manera inversamente proporcional con el grado de digestibilidad de la MS (Ramírez et al., 2002) y con el grado de degradabilidad de la pared celular (Traxler et al., 1998), siendo así los tratamientos con mayor contenido de lignina menos digestibles y su fibra menos aprovechable por el animal a nivel del rumen. En el caso de la adición de urea en las coronas de piña, esta investigación no se mostró diferencias en el contenido de lignina.

Al comparar la concentración de lignina de los tratamientos evaluados con los trabajos informados por Rogério et al. (2007) (10,05\% MS), Azevêdo et al. (2011) (3,71\% MS), Cunha et al. (2009) $(4,1-5,4 \%$ MS), Correia et al. (2006) (6,11\% MS) y los tratamientos con adición de pollinaza evaluados por Gutiérrez et al. (2003) (1,95 - 6,90\% MS), se determinó un valor menor, lo cual se podría relacionar con las características del material ensilado en los trabajos analizados. Los tratamientos con inclusión de heno presentaron valores similares a los descritos por López et al. (2009) $(2,46$ $2,93 \% \mathrm{MS})$; sin embargo, los tratamientos control y con $10 \%$ de inclusión de PCD mostraron valores menores, comportamientos que se podrían relacionar con el tipo de material secante adicionado.

Carbohidratos no fibrosos. Tanto la PCD como el heno afectaron el contenido de carbohidratos en los materiales ensilados. Cuando se utilizó PCD el contenido de CNF aumentó de forma significativa $(\mathrm{p}<0,05)$, mientras que al utilizar heno el contenido de esta fracción disminuyó de forma significativa $(p<0,05)$. Tendencias que se asocian a la concentración de $\mathrm{CNF}$ presentes en las materias primas utilizadas, como materiales absorbentes, alta en la PCD (RojasBourrillón et al., 2001; Villareal et al., 2006) y baja en el heno (WingChing y Alvarado, 2009).
Al utilizar la ecuación descrita por Detmann y Filho, (2010) se excluye el aporte de la urea en la PC para evitar subestimar el contenido de CNF de los materiales conservados. Al hacer esto se pudo determinar que la inclusión de urea no generó diferencias significativas entre los tratamientos evaluados (Cuadro 1).

Los resultados obtenidos en los tratamientos control y con inclusión de PCD fueron mayores al $6,59-13,6 \%$ reportados al utilizar rastrojos de piña ensilados con inclusión de melaza deshidratada con minerales y urea (Rodríguez-Chacón et al., 2014). No obstante, los resultados obtenidos fueron similares a los publicados por Rogério et al. (2007) (14,07\% MS) con plantas enteras, López et al. (2014) (4,58 - 17,7\% MS) con ensilajes de rastrojos de piña con urea, y por Azevêdo et al. (2011) (27,20\% MS) con rastrojos. Los valores de CNF de los tratamientos con heno fueron menores a los reportados por López et al. (2009) (23,5 - 37,2\% MS) con ensilados de rastrojos de piña con inclusión de PCD.

\section{Caracterización energética de los tratamientos ensilados}

Nutrimentos digestibles totales. Los nutrimentos digestiles totales no fueron afectados de forma negativa por el contenido de heno $(\mathrm{p}<0,05)$, al disminuir el aporte energético de los ensilados conforme se aumentó el contenido de este aditivo en la mezcla (Cuadro 2). Por el contrario, al incorporar PCD en la mezcla ensilada se incrementó el contenido energético de los ensilados con respecto a las coronas ensiladas sin aditivos (Cuadro 2), lo que generó una clara diferencia al comparar los materiales absorbentes, la cual podría estar limitada por el precio de cada una al momento de elaborar las mezclas. La incorporación de urea produjo diferencias entre el tratamientos control y los tratamientos con nivel de $1,5 \%$ de urea; sin embargo, no existieron diferencias entre los tratamientos con 0,5 y $1 \%$ de urea.

Los valores de NDT de los tratamientos fluctuaron entre 1,86 - 5,43 puntos porcentuales con respecto al tratamiento control, la diferencia fue mayor al control cuando se adicionó PCD a la mezcla, caso contrario, cuando se incorporó heno a la mezcla ensilada, los valores fueron menores al promedio del control cuando no se agregó urea a la mezcla, pero al agregar la urea no hubo diferencias con el control.

El contenido de NDT de los tratamientos con inclusión de PCD fue similar al valor obtenido por 
López et al. (2009) $(61,96$ - 67,14\%) con ensilados de rastrojos de piña con inclusión de PCD, mientras que los tratamientos con inclusión de heno fueron similares a los reportados por López et al. (2014) con rastrojos de piña con inclusión de urea.

Todos los tratamientos fueron mayores a los resultados publicados por Rogério et al. (2007) $(55,95 \%)$ con plantas enteras, por Otagaki et al. (1961) con heno de planta de piña $(46,9 \%)$ y pulpa de piña $(58,9 \%)$. También fueron mayores a los trabajos de Correia et al. (2006) $(57,81 \%)$ y Pereira et al. (2009) $(57,17 \%)$ con cáscaras y pulpa de piña. Además, fueron mayores a los resultados obtenidos por Betancourt (2004) $(60,91 \%)$ con ensilados de maíz. Los tratamientos con inclusión de PCD presentaron valores similares a los ensilados de maíz sin aditivos $(66,80 \%)$ (Cubero et al., 2010).

Energía neta de lactancia. Los tratamientos con mayor contenido de energía para la producción de leche, fueron en los que se utilizó la PCD como aditivo, en comparación con el tratamiento control. Se obtuvo una disminución en el contenido energético cuando se adicionó heno en la mezcla $(\mathrm{p}<0,05)$, comportamiento asociado a las características propias de cada material. La adición de urea a las mezclas generó una disminución en el contenido de nutrimentos digestibles totales a niveles de $1,5 \% \mathrm{p} / \mathrm{p}$, por lo que las fracciones energéticas sufren la misma tendencia, ya que se usó el contenido de NDT, para las estimaciones energéticas de los materiales (Cuadro 2).

Conforme aumentó el consumo de ensilado, aumentó la cantidad de leche producida (Figura 2), esto era esperable, debido a que hubo un mayor aporte de nutrimentos al animal para aumentar la producción. También se pudo observar una tendencia a la disminución del potencial productivo de los tratamientos con heno, debido a un menor contenido de energía en este aditivo (WingChing y Alvarado, 2009). Todos los tratamientos con aditivos, tuvieron más potencial para la producción de leche con respecto al tratamiento control. Cuando se utilizan en el mayor nivel de consumo (15 kg MV/vaca/día), los materiales ensilados permiten una producción de 3,5-4,5 kg de leche/vaca/día.

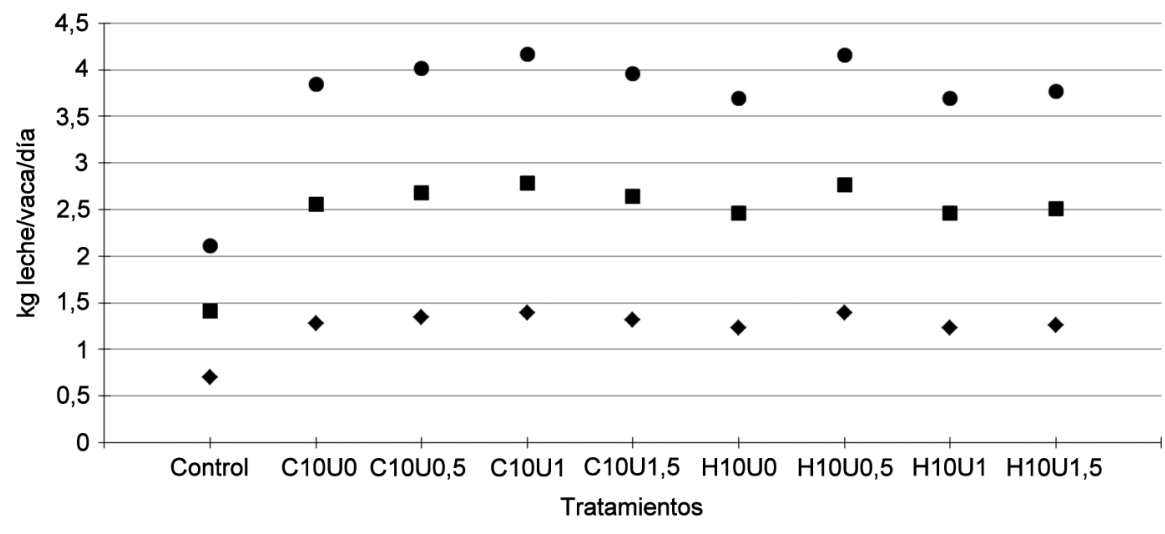

- Consumo 5 kg MV/vaca/día —Consumo 10 kg/vaca/día •Consumo 15 kg MV/vaca/día

Figura 2. Potencial de producción por la energía neta de lactación $\left(\mathrm{EN}_{\mathrm{L}}\right)$, de los ensilados de corona de piña con pulpa de cítricos deshidratada $(\mathrm{C})$, heno $(\mathrm{H})$ y urea $(\mathrm{U})$ de acuerdo al nivel de consumo de materia verde (MV) (5, 10 y 15 kg MV/día). San José, Costa Rica. 2014.

Figure 2. Potential production by net energy value for lactation (by $\mathrm{NE}_{\mathrm{L}}$ requirement) in $\mathrm{kg}$ of milk/cow/day, from pineapple crown silage with dehydrated citrus pulp (C), hay (H), and urea (U), according to fresh matter (FM) intake (5, 10 y $15 \mathrm{~kg} \mathrm{FM/day).} \mathrm{San} \mathrm{José,}$ Costa Rica. 2014. 


\section{LITERATURA CITADA}

AOAC (Association of Official Analytical Chemist). 1998. Official Methods of Analysis of AOAC International. 16th ed, 4th rev. Gaithersburg, MD, USA.

Arroyo, C., A. Rojas-Bourrillon, y R. Rosales. 2003. Uso de urea o pollinaza como suplemento proteico para toretes consumiendo ensilaje de pulpa de pejibaye. Agron. Costarricense 27(2):69-73.

Azevêdo, J.A., S.C. Filho, E. Detmann, D. Pina, L.G. Pereira K. De Oliveira, H. Fernandes, e N.K. Souza. 2011. Predição de frações digestíveis e valor energético de subprodutos agrícolas e agroindustriais para bovinos. Rev. Bras. Zootec. 40:391-402.

Bartholomew, D.P., R.E. Paull, and KG. Rohrbach. 2003. Pineapple: botany and uses. Cabi Publishing, GBR.

Belyea, R., B. Steevens, G. Garner, J. Whittier, and H. Sewell. 1996. Using NDF and ADF to balance diets. Missouri University Extension: g3161. MO, USA.

Betancourt, J.C. 2004. Caracterización nutricional y productiva de material fresco y ensilado de maní forrajero (Arachis pintoi) cultivado en asocio con maíz (Zea mays), a tres densidades de siembra. Tesis M.Sc., Universidad de Costa Rica, San José, CRC.

Correia, M.X., R.G. Costa, J.H. Da Silva, F.F. De Carvalho, e A. De Medeiros. 2006. Utilização de resíduo agroindustrial de abacaxi desidratado em dietas para caprinos em crescimento: digestibilidade e desempenho. Rev. Bras. Zootec. 35:1822-1828.

Cubero, J.F., A. Rojas, y R. WingChing. 2010. Uso del inóculo microbial elaborado en finca en ensilaje de maíz (Zea mays). Valor nutricional y fermentativo. Agron. Costarricense 34(2):237-250.

Cunha, M.D.G., E. Oliveira, J.L. Ramos, e M.D. Alcântara. 2009. Conservação e utilização do resíduo de abacaxi na alimentação de ovinos no curimataú ocidental da paraíba. Rev. Tecnol. Ciênc. Agropec. 3(3):55-62.

Detmann, E., e S.C. Filho. 2010. Sobre a estimação de carboidratos não fibrosos em alimentos e dietas. Arq. Bras. Med. Vet. Zootec. 62:980-984.

Di Rienzo, J.A., F. Casanoves, M.G. Balzarini, L. Gonzalez M. Tablada, y Y.C. Robledo. 2013. InfoStat versión 2013. Grupo InfoStat, FCA, Universidad Nacional de Córdoba, ARG.

Gutiérrez, F., A. Rojas, H. Dormond, M. Poore, y R. WingChing. 2003. Características nutricionales y fermentativas de mezclas ensiladas de desechos de piña y avícolas. Agron. Costarricense 27(1):79-89.
Kellems, R.O., O. Wayman, A.H. Nguyen, J.C. Nolan, C.M. Campbell, J.R. Carpenter, and E.B. Ho-A. 1979. Postharvest pineapple plant forage as a potential feedstuff for beef cattle: evaluated by laboratory analyses in vitro and in vivo digestibility and feedlot trials. J. Anim. Sci. 48:1040-1048.

Lallo, F.H., I. Nunes, W. Gonçalves, L.M. Zeoula, F. Barros, e F. Yoshimi. 2003. Níveis de substitução da silagem de milho pela silagem de resíduos industriais de abacaxi sobre a degradabilidade ruminal em bovinos de corte. Rev. Bras. Zootec. 32:719-726.

Licitra, G., T.M. Hernandez, and P.J. Van Soest. 1996. Standardization of procedures for nitrogen fractionation of ruminant feeds. Anim. Feed Sci. Technol. 57:347-358.

López, M., R. WingChing-Jones, y A. Rojas. 2009. Características fermentativas y nutricionales del ensilaje de rastrojo de piña (Ananas comosus). Agron. Costarricense 33(1):1-15.

López, M., R. WingChing-Jones, A. Rojas, y S. Rodríguez. 2014. Valor nutricional del ensilaje de rastrojo de piña con niveles crecientes de urea. Nutrición Animal Tropical 8(1):1-20

Lousada Jr.. J.E, J. Miranda, N. Rodríguez, J.C. Machado, e R. Braga. 2005. Consumo e digestibilidade de subprodutos do processamento de frutas em ovinos. Rev. Bras. Zootec. 34:659-669.

McDonald, P. 1981. The biochemistry of silage. John Wiley Ltd. NY, USA.

NRC (National Research Council). 2001. Nutrient requirements of dairy cattle. 7 th ed. National Academy Press, WA, USA.

Otagaki, K., G.P. Lofgreen, and E. Cobb. 1961. Net energy of pineapple bran and pineapple hay fed to lactating dairy cows. J. Dairy Sci. 44:491-497.

Pereira, E.S., J.G. Filho, E.R. Freitas, J.N. Neiva, e M.J. Cândido. 2009. Valor energético de subprodutos da agroindústria brasileira. Arch. Zootec. 58:455-458.

Prado, I., F.H. Lallo, L.M. Zeoula, S. Neto, W. Gonçalves, e J. Marques. 2003. Níveis de substitução da silagem de milho pela silagem de resíduos industriais de abacaxi sobre el desempenho de bovinos confinados. Rev. Bras. Zootec. 32:737-744.

Ramírez, R., R.G. Ramírez, y F. López. 2002. Factores estructurales de la pared celular del forraje que afectan su digestibilidad. Ciencia UANL 2:180-189.

Rebolledo, A., A.L. Pérez, L. Rebolledo, y A.E. Becerril. 2006. Rendimiento y calidad de fruto de cultivares 
de piña en densidades de plantación. Rev. Fitotec.Mex. 29:55-62.

Rodríguez-Chacón, S., M. López-Herrera, R. WingChing, y A. Rojas-Bourrillon. 2014. Adición de melaza deshidratada y urea en ensilados de rastrojos de piña Agron. Mesoam. 25:313-321.

Rogério, M.C.P., I. Borges, J.N.M. Neiva, N.M. Rodriguez, J.C.M. Pimentel, G.A. Martins, T.P. Ribeiro, J.B. Costa, S.F. Santos, e F.C. Carvalho. 2007. Valor nutritivo do resíduo da indústria processadora de abacaxi (Ananas comosus L.) em dietas para ovinos. 1. Consumo, digestibilidade aparente e balanços energético e nitrogenado. Arq. Bras. Med. Vet. Zootec. 59:773-781.

Rojas-Bourrillón, A., L. Gamboa, M. Villareal, E. Víquez R. Castro, y M. Poore. 2001. La sustitución de maíz por pulpa de cítricos deshidratada sobre la producción y composición láctea de vacas encastadas Holstein en el trópico húmedo de Costa Rica. Agron. Costarricense 25(1):45-52.

Sánchez, J. 2010. Práctica en el Programa de Transferencia Tecnológica de la Cooperativa de Productores de Leche Dos Pinos R.L. Ciudad Quesada, San Carlos. Práctica Bach, Universidad de Costa Rica, San José, CRC.

Sánchez, J.M., y H. Soto. 1998. Estimación de la calidad nutricional de los forrajes del cantón de San Carlos. II. Componentes de la pared celular. Nutrición Animal Tropical 4(1):7-19.
Traxler, M.J., D.G. Fox, P.J. Van Soest, A.N. Pell, C.E. Lascano, D.P. Lanna, J.E. Moore, R.P. Lana, M. Velez, and A. Flores. 1998. Predicting forage indigestible ndf from lignin concentration. J. Anim. Sci. 76:1469-1480.

Van Soest, P.J., and J.B. Robertson. 1985. Analysis of forages and fibrous food. As 613. Cornell University, a laboratory manual. Department of Animal Science, Ithaca, NY, USA.

Van Soest, P.V., J.B. Robertson, and B.A. Lewis. 1991. Methods for dietary fiber, neutral detergent fiber, and nonstarch polysaccharides in relation to animal nutrition. J. Dairy Sci. 74:3583-3597.

Villarreal, M., R.C. Cochran, A. Rojas-Bourrillón, O. Murillo, H. Muñoz, and M. Poore. 2006. Effect of supplementation with pelleted citrus pulp on digestibility and intake in beef cattle fed a tropical grass-based diet (Cynodon nlemfuensis). Anim. Feed Sci. Technol. 125:163-173.

Weiss, W.P. 2004. Fine-tuning energy calculations. In: M. Eastridge, editor, Proceedings of the Tri-State Dairy Nutrition Conference. Purdue University, Michigan State University, Ohio State University. April 27 - 28. Fort Wayne, IN, USA. p. 131-142.

WingChing, R., y G. Alvarado. 2009.Valor nutricional del heno de Transvala inoculado con el hongo Pleurotus ostreatus sp. Agron. Costarricense 33(1):147-153. 
